\title{
Enhanced cooling of hydrogen by a buffer gas of alkali-metal atoms
}

\section{Citation}

Derevianko, A., R. Côté, A. Dalgarno, and G.-H. Jeung. 2001. “Enhanced Cooling of Hydrogen by a Buffer Gas of Alkali-Metal Atoms." Physical Review A 64 (1). https://doi.org/10.1103/ physreva.64.011404.

\section{Permanent link}

http://nrs.harvard.edu/urn-3:HUL.InstRepos:41417411

\section{Terms of Use}

This article was downloaded from Harvard University's DASH repository, and is made available under the terms and conditions applicable to Other Posted Material, as set forth at http:// nrs.harvard.edu/urn-3:HUL.InstRepos:dash.current.terms-of-use\#LAA

\section{Share Your Story}

The Harvard community has made this article openly available.

Please share how this access benefits you. Submit a story. 


\title{
Enhanced cooling of hydrogen by a buffer gas of alkali-metal atoms
}

\author{
A. Derevianko, ${ }^{*}$ R. Côté ${ }^{\dagger}$ A. Dalgarno, and G.-H. Jeung ${ }^{\ddagger}$ \\ Institute for Theoretical Atomic and Molecular Physics, Harvard-Smithsonian Center for Astrophysics, Cambridge, Massachusetts 02138
}

(Received 22 January 2001; published 12 June 2001)

\begin{abstract}
We consider the possibility of enhanced cooling of hydrogen atoms by a buffer gas of alkali-metal atoms $\mathrm{Na}$, $\mathrm{K}, \mathrm{Rb}$, and $\mathrm{Cs}$. Ultracold elastic collision cross sections for the $\mathrm{Na}-\mathrm{H}$ and $\mathrm{Rb}-\mathrm{H}$ purely spin-polarized pairs are found to be 640 and 860 times larger than that for the $\mathrm{H}-\mathrm{H}$ pair, respectively. From an analysis of the techniques of production of ultracold sodium and rubidium samples, it seems feasible that the critical conditions for Bose-Einstein condensation of hydrogen could be achieved already at the stage of optical cooling of the sodium or rubidium buffer gas.
\end{abstract}

DOI: 10.1103/PhysRevA.64.011404

PACS number(s): 32.80.Pj, 31.15.Ar, 32.80.Cy, 34.20.-b

Bose-Einstein condensation (BEC) of a dilute gas of hydrogen [1] is a delicate experimental task because of the smallness of elastic cross sections, which impedes evaporative cooling. Côté et al. [2] have investigated the possibility of an enhanced cooling of atomic hydrogen by mixing with ultracold lithium gas. They found that at ultracold temperatures the ${ }^{7} \mathrm{Li}-\mathrm{H}$ elastic cross section of purely spin-polarized pairs is 1400 times larger than for $\mathrm{H}-\mathrm{H}$ collisions and therefore lithium could be efficient in accelerating the cooling of hydrogen. In the present work, we extend the treatment of Ref. [2] of the cooling of hydrogen in a buffer gas of lithium atoms to heavier $\mathrm{Na}, \mathrm{K}, \mathrm{Rb}$, and $\mathrm{Cs}$ atoms. There is a particular interest in ${ }^{23} \mathrm{Na}$ and ${ }^{87} \mathrm{Rb}$ stemming from the availability of established methods to efficiently create ultracold samples and Bose-Einstein condensates [3,4]. By a direct calculation, we will demonstrate that the ultracold $\mathrm{Na}-\mathrm{H}$ and $\mathrm{Rb}-\mathrm{H}$ elastic cross section are a factor of 640 and 860 larger than that for a pair of hydrogen atoms, respectively. We find that with available techniques for creating ultracold sodium (rubidium) samples, the critical BEC conditions for hydrogen could be achieved through laser cooling of the alkalimetal component, without resorting to techniques of cryogenic and evaporative cooling.

In Ref. [5] we presented quantum-mechanical calculations of the potential-energy curves for the singlet and triplet states of $\mathrm{LiH}, \mathrm{NaH}, \mathrm{KH}, \mathrm{RbH}$, and $\mathrm{CsH}$ formed by the approach of ground-state alkali-metal atoms and hydrogen atoms. We determined precise values for the coefficients of the van der Waals interaction and estimated the contribution of the exchange interaction at large distances. Together with empirical data, these were used to assess and improve the accuracy of the $a b$ initio potentials.

In a magnetic trap, atoms are confined in a "low-field seeking" spin-polarized state, and most ground-state collisions occur in the triplet molecular potential $a^{3} \Sigma^{+}$. In Fig. 1 we present the $a^{3} \Sigma^{+}$molecular potentials between hydrogen

\footnotetext{
*Permanent address: Department of Physics, University of Nevada, Reno, NV 89557.

${ }^{\dagger}$ Permanent address: Department of Physics, University of Connecticut, Storrs, CT 06269-3046.

*Permanent address: Laboratoire Aimé Cotton (CNRS UPR3321), Bâtiment 505, Université de Paris Sud, 91405 Orsay, France.
}

and alkali-metal atoms, constructed in Ref. [5]. The potential wells at large internuclear distances are formed due to the interplay of repulsive exchange interaction and attractive van der Waals forces. Although at a given internuclear separation both interactions are stronger for a heavier dimer [5], the position of the minimum shifts towards larger distances as the mass of the alkali-metal atom increases. The potential well is deeper for lighter dimers. Each of these wells supports a single bound vibrational state. From the numerical solution of the eigenvalue problem, we determined the positions of the bound states and we present them in Table I.

Magnetic trap losses occur if during a collision the atom changes the projection of its spin onto the confining magnetic field. These spin-flip collisions are characterized by both singlet $X^{1} \Sigma^{+}$and triplet $a^{3} \Sigma^{+}$potentials. The singlet potentials are presented in Fig. 2. The $X^{1} \Sigma^{+}$potential wells are substantially deeper than the $a^{3} \Sigma^{+}$triplet potentials. These wells are formed by an interplay between repulsive short-range forces and attractive exchange and van der Waals interactions. Since the atomic core is bigger for heavier alkali-metal atoms, the minima of the potentials are located at increasingly larger internuclear distances from sodium hydride to cesium hydride. The singlet potentials, presented in Fig. 2, were constructed in Ref. [5] by combining the empirical results, reviewed in Ref. [6], with those from molecular-structure calculations. The $a b$ initio dissociation energies for the $X^{1} \Sigma^{+}$states agree to within $2 \%$ with experimental values. Contrary to the $X^{1} \Sigma^{+}$case, there are no empirical data to assess the quality of the $a^{3} \Sigma^{+}$potentials. We assume that the adopted $a^{3} \Sigma^{+}$potentials have an accu-

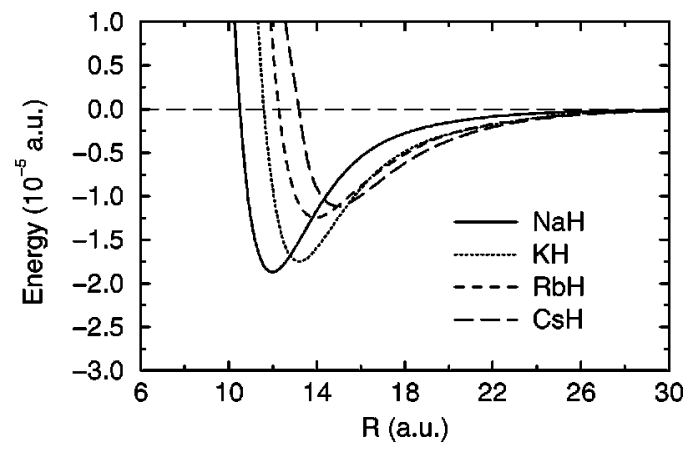

FIG. 1. $a^{3} \Sigma^{+}$potentials for alkali hydrides [5]. 
TABLE I. Binding energy of the vibrational state supported by $a^{3} \Sigma^{+}$potentials and the scattering lengths calculated in the effective-range approximation, Eq. (3). All values are in atomic units.

\begin{tabular}{lcccc}
\hline \hline & ${ }^{23} \mathrm{Na}-\mathrm{H}$ & ${ }^{39} \mathrm{~K}-\mathrm{H}$ & ${ }^{87} \mathrm{Rb}-\mathrm{H}$ & ${ }^{133} \mathrm{Cs}-\mathrm{H}$ \\
\hline$E_{b}$ & $2.964 \times 10^{-7}$ & $7.334 \times 10^{-7}$ & $2.443 \times 10^{-7}$ & $1.785 \times 10^{-7}$ \\
$a_{T}^{\text {eff }}$ & 31.0 & 19.5 & 33.6 & 39.2 \\
\hline \hline
\end{tabular}

racy of the order of $1 \%$, based on the agreement of theory and experiment for the singlet potentials [5].

Using the constructed potentials [5], we calculated radial continuum wave functions using the Numerov method, and obtained phase shifts $\delta_{l}$, from which we determined scattering lengths, cross sections, and rate coefficients. The singlet and triplet scattering lengths $a_{S}$ and $a_{T}$ were calculated by taking the low-energy limit

$$
a=-\lim _{k \rightarrow 0} \frac{\tan \delta_{0}(k)}{k},
$$

where $\delta_{0}$ is the $s$-wave phase shift, and $k$ is the wave vector of relative motion. The values of $a_{S}$ and $a_{T}$ are listed in Table II. We tested the sensitivity of the scattering lengths by performing calculations with uniformly scaled potentials

$$
U(R) \rightarrow(1+\lambda) U(R)
$$

The scattering lengths corresponding to $\lambda= \pm 1 \%$ are presented in Table II. The triplet scattering lengths are insensitive to such modifications, because the shallow $a^{3} \Sigma^{+}$potentials each support a single bound state. Due to the large density of bound vibrational states in the $X^{1} \Sigma^{+}$potential, the singlet scattering length $a_{S}$ is very sensitive to uncertainties in the potential. Indeed, deepening the reference potential by $1 \%$ for $\mathrm{KH}$ allows a new bound state to appear, causing $a_{S}$ to pass through infinitely large values.

We may estimate the scattering length from effective range theory as

$$
a^{\mathrm{eff}} \approx \frac{\hbar}{\sqrt{2 \mu E_{b}}}
$$

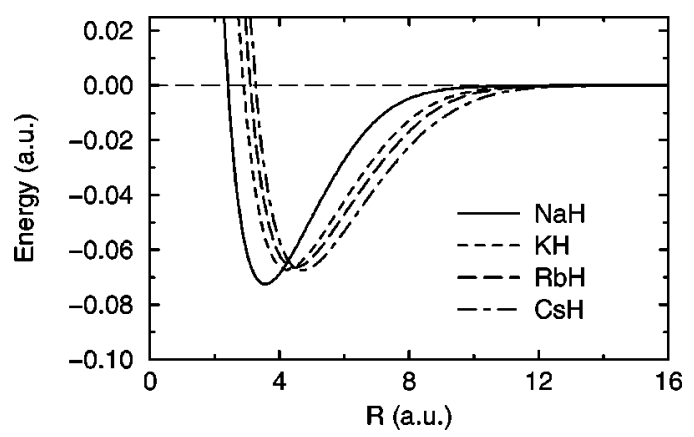

FIG. 2. $X^{1} \Sigma^{+}$potentials for alkali hydrides [5].
TABLE II. Scattering lengths in a.u. for $a^{3} \Sigma^{+}$and $X^{1} \Sigma^{+}$potentials. The entries marked hybrid were calculated employing potentials constructed in Ref. [5]. Other values were obtained with potentials uniformly scaled as $U(R) \rightarrow \lambda U(R)$.

\begin{tabular}{lcccc}
\hline \hline$\lambda$ & ${ }^{23} \mathrm{Na}-\mathrm{H}$ & ${ }^{39} \mathrm{~K}-\mathrm{H}$ & ${ }^{87} \mathrm{Rb}-\mathrm{H}$ & ${ }^{133} \mathrm{Cs}-\mathrm{H}$ \\
\hline \multirow{5}{c}{$a^{3} \Sigma^{+}$} \\
hybrid & 43.2 & 34.7 & 50.2 & 56.8 \\
$-1 \%$ & 44.3 & 35.2 & 51.4 & 58.4 \\
$+1 \%$ & 42.3 & 34.3 & 49.1 & 55.4 \\
\multicolumn{5}{c}{} \\
hybrid & -9.77 & -92.6 & 1.07 & 9.78 \\
$-1 \%$ & 0.73 & -2.99 & 7.42 & 14.5 \\
$+1 \%$ & -83.6 & 41.9 & -11.0 & 4.22 \\
\hline \hline
\end{tabular}

where $E_{b}$ is the binding energy of the last bound state. The resulting scattering lengths are presented in Table I; they are in fair agreement with the results of the direct calculations listed in Table II.

The total elastic cross section is expressed through the phase shifts as

$$
\sigma=\frac{4 \pi}{k^{2}} \sum_{l=0}(2 l+1) \sin ^{2} \delta_{l},
$$

while the transport (momentum-transfer) cross section is calculated from

$$
\begin{aligned}
\sigma_{\mathrm{tr}} & =\int(1-\cos \theta) d \sigma, \\
& =\frac{4 \pi}{k^{2}} \sum_{l=0}(l+1) \sin ^{2}\left(\delta_{l+1}-\delta_{l}\right) .
\end{aligned}
$$

The results are shown in Fig. 3. For ultracold temperatures, where $s$-wave scattering dominates, the total and the transport cross section are equal and are given by $4 \pi a^{2}$. The $l=1$ partial waves start contributing at the $1 \%$ level when energies of $8 \times 10^{-8}$ hartree are reached for $\mathrm{NaH}$.

Assuming equal temperatures of the hydrogen and alkalimetal gases, we determine rate coefficients for elastic scattering as

$$
k_{r}=\sqrt{\frac{8}{\pi \mu \beta}} \int_{0}^{\infty}(\beta E) \sigma(E) e^{-\beta E} d(\beta E),
$$

where $\beta \equiv 1 /\left(k_{B} T\right), E=\hbar^{2} k^{2} /(2 \mu)$, and $\mu$ is the reduced mass of the system. The results of the calculation are presented in Fig. 4. For temperatures below $1 \mathrm{mK}$, the rate coefficients are accurately approximated by $k_{r}$ $=4 \pi a^{2} \sqrt{8 / \pi \mu \beta}$, where $a$ is the relevant scattering length.

In experiments on Bose-Einstein condensation [1], a cloud of hydrogen atoms is placed in a magnetic field, which traps spin-polarized low-field seeking atoms. The corresponding triplet scattering length [7] is $a_{T}^{\mathrm{H}-\mathrm{H}}=1.212 a_{0}\left(a_{0}\right.$ : bohr radius). Our calculations demonstrate that the triplet 


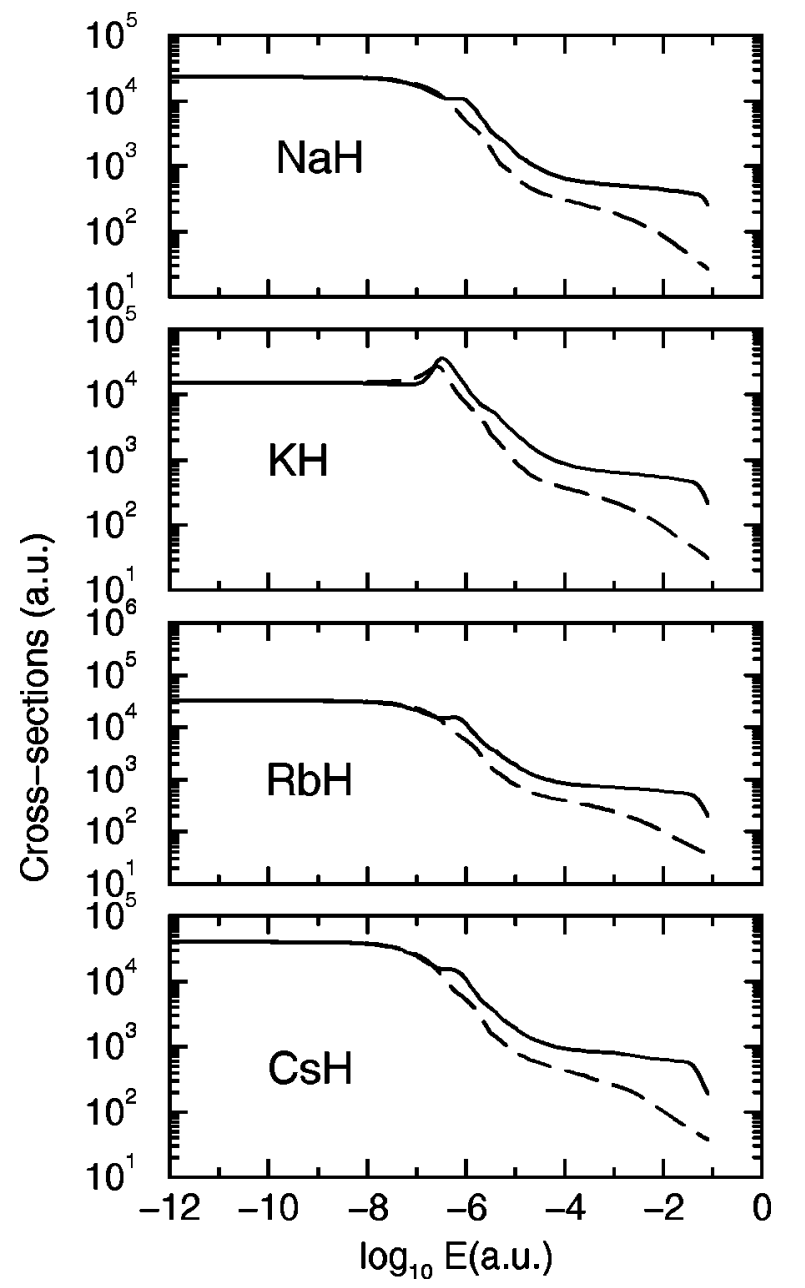

FIG. 3. Elastic (solid lines) and transport (dashed lines) cross sections for collision of spin-polarized alkali-metal atoms and hydrogen.

elastic cross sections for the sodium-hydrogen pair ( $\sigma$ $=4 \pi a^{2}$ ) are 640 times larger than that for the hydrogenhydrogen pair $\left(\sigma=8 \pi a^{2}\right)$. For the rubidium-hydrogen pair, this factor is 860 . The efficiency of the kinetic-energy transfer to the hydrogen atom is proportional to the ratio of the mass of the hydrogen atom to the mass of the alkali-metal atom A. Accordingly we define as a measure of the thermalization efficiency of a single collision of a hydrogen atom with a buffer-gas atom the ratio

$$
f=\frac{M_{\mathrm{H}}}{M_{\mathrm{A}}} \frac{\sigma(\mathrm{A}-\mathrm{H})}{\sigma(\mathrm{H}-\mathrm{H})}=\frac{1}{2} \frac{M_{\mathrm{H}}}{M_{\mathrm{A}}}\left(\frac{a_{T}^{\mathrm{A}-\mathrm{H}}}{a_{T}^{\mathrm{H}-\mathrm{H}}}\right)^{2} .
$$

The calculated values are listed in Table III. We include ${ }^{7} \mathrm{Li}$ for which the triplet scattering length [2] is $a_{T}=65(5) a_{0}$. Due to its smaller mass, ${ }^{7} \mathrm{Li}$ may be the best candidate for a buffer-gas cooling technique with an efficiency factor $f$ $=205$. Sodium has $f=28 ; \mathrm{K}, \mathrm{Rb}$, and $\mathrm{Cs}$ are less efficient with $f \approx 10$.

Spin-flip collisions lead to trap losses. In the limit of small energies of relative motion, the corresponding cross section is estimated by $\sigma_{\mathrm{SF}}=\pi\left(a_{T}-a_{S}\right)^{2}$ [8]. Using scatter-

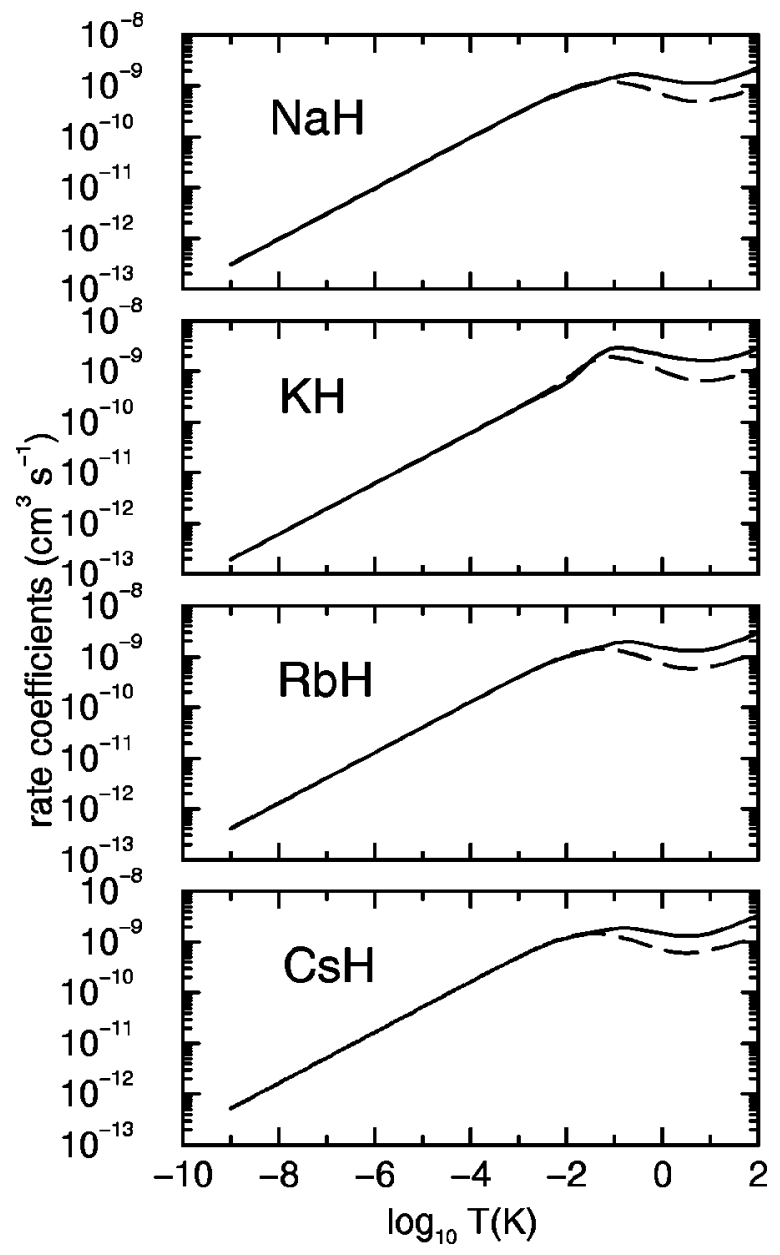

FIG. 4. Elastic (solid lines) and momentum-transfer (dashed lines) rate coefficients for collisions of spin-polarized alkali-metal atoms and hydrogen.

ing lengths calculated with the reference potentials, we find for $\mathrm{NaH} \sigma_{\mathrm{SF}} \approx 8800$ a.u. Unfortunately, due to the large uncertainties in the singlet scattering lengths, no definite conclusions can be drawn about spin-flip losses, except that they are probably large if collisions are not in a pure triplet state.

In the hydrogen BEC experiment [1], the Ioffe-Pritchard magnetic trap is loaded with hydrogen atoms at $T$ $\approx 250 \mathrm{mK}$. Further cooling is achieved by contact of the atoms with a cryogenic wall. The large frequency of the Ly$\alpha$ transition prevents the use of optical cooling for hydrogen. As an alternative, an alkali-metal buffer gas could be introduced and laser cooled driving down the temperature of the hydrogen component through collisions with alkali atoms. At this active stage the temperature of the combined system could be potentially lowered to $50-100 \mu \mathrm{K}$ (e.g., as in the recent MIT experiment [4] on sodium BEC). The critical

TABLE III. Measure of efficiency $f$, Eq. (8), of cooling of a hydrogen sample by a buffer gas of alkali-metal atoms.

\begin{tabular}{ccccc}
\hline \hline${ }^{7} \mathrm{Li}$ & ${ }^{23} \mathrm{Na}$ & ${ }^{39} \mathrm{~K}$ & ${ }^{87} \mathrm{Rb}$ & ${ }^{133} \mathrm{Cs}$ \\
\hline 205 & 28 & 11 & 10 & 8 \\
\hline \hline
\end{tabular}


temperature of the hydrogen BEC transition is $50 \mu \mathrm{K}[1]$. Therefore, depending on the densities, the BEC conditions for the hydrogen component could be achieved at the optical stage. In contrast, temperatures no lower than $200 \mu \mathrm{K}$ have been obtained for Li [9]. Although Li $(f=205)$ is more efficient than $\mathrm{Na}(F=28)$ at cooling $\mathrm{H}$, the critical temperature cannot be reached by optical cooling using Li.

In the subsequent passive scheme both components could be evaporatively cooled simultaneously. The evaporative cooling of a hydrogen cloud is very slow due to the small $\mathrm{H}-\mathrm{H}$ elastic cross sections. In the combined system with $\mathrm{Na}$ as the buffer gas, the sodium atoms would serve as a source of thermalization for hydrogen. The efficiency of thermalization is determined by both cross sections and the relative densities of two gases. Peak densities of sodium BEC with $n_{p} \approx 1.5 \times 10^{14} \mathrm{~cm}^{-3}$ comparable to densities of hydrogen [1] were recently reported [3] (similarly, Rb could also be used, although momentum transfer is less efficient). How- ever, superfluidity properties of a sodium (or rubidium) condensate may limit the efficiency of sympathetic cooling of hydrogen at extremely low temperatures [10].

To summarize, an efficient pathway for achieving BoseEinstein condensation of hydrogen through enhanced cooling by a buffer gas of sodium atoms seems feasible, due to large elastic cross sections for sodium-hydrogen collisions. We emphasize that it may be possible to optically cool the combined system to the critical conditions required for a hydrogen BEC.

The work of A.D. and A.D. was supported by the Chemical Sciences, Geosciences and Biosciences Division of the Office of Basic Energy Sciences, Office of Science, U.S. Department of Energy. G.-H.J. was partially supported by the Centre National de la Recherche Scientifique (CNRS), and R.C. by the National Science Foundation, Grant No. PHY-9970757. The authors would like to thank Vasili Kharchenko for useful discussions.
[1] D.G. Fried et al., Phys. Rev. Lett. 81, 3811 (1998); T.C. Killian et al., ibid. 81, 3807 (1998).

[2] R. Côté, M.J. Jamieson, Z-C. Yan, N. Geum, G.-H. Jeung, and A. Dalgarno, Phys. Rev. Lett. 84, 2806 (2000).

[3] C. Raman, M. Köhl, R. Onofrio, D.S. Durfee, C.E. Kuklewicz, Z. Hadzibabic, and W. Ketterle, Phys. Rev. Lett. 83, 2502 (1999).

[4] R. Onofrio, D.S. Durfee, C. Raman, M. Köhl, C.E. Kuklewicz, and W. Ketterle, Phys. Rev. Lett. 84, 810 (2000).

[5] N. Geum, G.-H. Jeung, A. Derevianko, R. Côté, and A. Dalgarno, J. Chem. Phys. (to be published).
[6] W. Stwalley, W. Zemke, and S.C. Yang, J. Phys. Chem. Ref. Data 20, 153 (1991).

[7] M.J. Jamieson, A. Dalgarno, and M. Kimura, Phys. Rev. A 51, 2626 (1995).

[8] A. Dalgarno and M.R.H. Rudge, Proc. R. Soc. London, Ser. A 286, 519 (1965); G.M. Carter and D.E. Pritchard, Phys. Rev. A 5, 1225 (1972).

[9] C.C. Bradley, C.A. Sackett, and R.G. Hulet, Phys. Rev. Lett. 78, 985 (1997).

[10] E. Timmermans and R. Côté, Phys. Rev. Lett. 80, 3419 (1998); A.P. Chikkatur, ibid. 85, 483 (2000). 